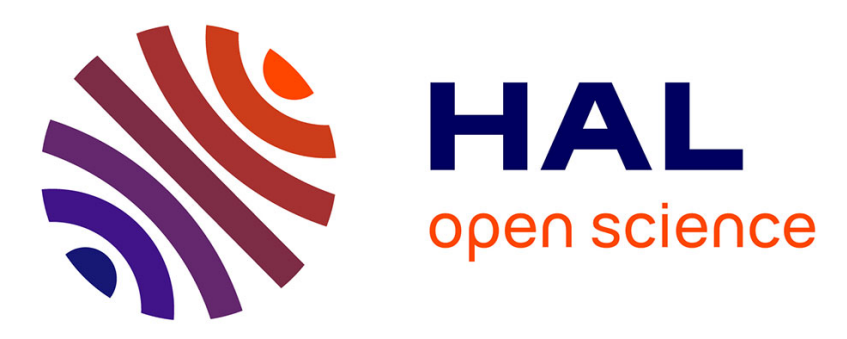

\title{
Drag reduction of a 3D bluff body using coherent streamwise streaks
}

\author{
Grégory Pujals, Sébastien Depardon, Carlo Cossu
}

\section{To cite this version:}

Grégory Pujals, Sébastien Depardon, Carlo Cossu. Drag reduction of a 3D bluff body using coherent streamwise streaks. Experiments in Fluids, 2010, 49 (5), pp.1085-1094. 10.1007/s00348-010-0857-5 . hal-01020631

\section{HAL Id: hal-01020631 \\ https://hal-polytechnique.archives-ouvertes.fr/hal-01020631}

Submitted on 7 Dec 2017

HAL is a multi-disciplinary open access archive for the deposit and dissemination of scientific research documents, whether they are published or not. The documents may come from teaching and research institutions in France or abroad, or from public or private research centers.
L'archive ouverte pluridisciplinaire HAL, est destinée au dépôt et à la diffusion de documents scientifiques de niveau recherche, publiés ou non, émanant des établissements d'enseignement et de recherche français ou étrangers, des laboratoires publics ou privés. 


\title{
Drag reduction of a 3D bluff body using coherent streamwise streaks
}

\author{
G. Pujals $\cdot$ S. Depardon $\cdot$ C. Cossu
}

\begin{abstract}
Separation on the rear-end of an Ahmed body is suppressed by means of large-scale coherent streaks forced on the roof of the model. These streaks originate from an array of suitably shaped cylindrical roughness elements and are amplified by the mean shear through the lift-up effect. Interacting with the mean velocity field at leading order, they induce a strong controlled spanwise modulation. The resulting streaky base flow is observed to sustain the adverse pressure gradient since PIV measurements as well as static wall pressure distributions show that the recirculation bubble completely vanishes. These modifications of the topology of the flow are associated with a substantial drag reduction, which can be of about $10 \%$ when the roughness array is optimally placed on the roof of the bluff body.
\end{abstract}

G. Pujals · S. Depardon

PSA Peugeot Citroën, Centre Technique de Vélizy,

2 Route de Gisy, 78943 Vélizy-Villacoublay Cedex, France

G. Pujals $(\bowtie) \cdot$ C. Cossu

LadHyX, CNRS-École Polytechnique, 91128 Palaiseau, France

e-mail: gregory.pujals@ladhyx.polytechnique.fr

Present Address:

G. Pujals

Institut Français du Pétrole, 1-4 av. de bois Préau,

92852 Rueil-Malmaison, France

Present Address:

C. Cossu $(\bowtie)$

IMFT-CNRS, allée du Pr. Camille Soula,

31400 Toulouse, France

e-mail: carlo.cossu@imft.fr

\section{Introduction}

Boundary layer separation occurs when low momentum flow (i.e. in the near-wall region of a boundary layer) faces an adverse pressure gradient which tends to lower its motion. This pressure gradient could be due to geometrical constraints (rear-end of a ground vehicle) or operating conditions (airfoil at high angle of attack as it could happen during take-off or landing). In many industrial applications, boundary layer separation is associated with a large loss of performance (increase in fuel consumption in the case of ground vehicles, decrease in the lift-force on airplanes' wing at high angle of attack) making separation control of great importance.

\subsection{Separation control with vortex generators}

One obvious way to delay or even suppress separation is to bring momentum into the near-wall region of the boundary layer. Since the flow in this region is less energetic, this enhancement of momentum would enable it to sustain the pressure gradient. Active closed-loop flow control has proved to be effective in reducing the drag of bluff bodies [see e.g. Pastoor et al. (2008) where a $20 \%$ drag reduction is achieved on a 2D bluff body with blunt rear-end]. Nevertheless, passive (open-loop) separation control is very attractive because, as it is easier to implement and to handle, it has very low production and maintenance costs. The most widely used passive devices are vortex generators (VGs). Introduced in the late forties by Taylor (1947), the early VGs consist in arrays of small vanes which height $k$ is comparable to the undisturbed boundary layer thickness $\delta_{0}$ at the same position. In the late 1980s, the work of Rao and Kariya (1988) suggested that submerged VGs (i.e. $\left.k / \delta_{0} \leq 0.6\right)$ can be even more efficient than the classical 
Table 1 Most effective parameters used in previous studies involving VGs

\begin{tabular}{|c|c|c|c|c|c|c|}
\hline Investigator(s) & Model used & VGs & $k / \delta_{0}$ & $\lambda_{z} / \delta_{0}$ & $l / k\left(l / \lambda_{z}\right)$ & $x_{V G} / k\left(x_{V G} / \lambda_{z}\right)$ \\
\hline Lin (1999) & 2D backward-facing ramp & $\mathrm{CtR}$ rectangular vanes & 0.2 & 1.8 & $2.0(0.22)$ & $10(1.1)$ \\
\hline Betterton et al. (2000) & 2D bump & CtR triangular vanes & 0.3 & 3.6 & $6.0(0.5)$ & $52(4.3)$ \\
\hline Godard and Stanislas (2006) & 2D bump & CtR triangular vanes & 0.37 & 2.2 & $2.5(0.4)$ & $57(9.5)$ \\
\hline Duriez et al. (2006) & 2D backward-facing ramp & CtR cylindrical elements & 0.6 & 2.4 & $1.3(0.33)$ & $13.3(4.44)$ \\
\hline Angele and Grewe (2007) & Flat plate with suction & $\mathrm{CtR}$ rectangular vanes & 1.0 & 10.3 & $2.5(0.24)$ & $16(1.55)$ \\
\hline Park et al. (2007) & 2D backward-facing step & Rectangular tabs & 0.5 & 4.0 & $1.0(0.12)$ & $0.0(0.0)$ \\
\hline
\end{tabular}

VGs of size $\approx \delta_{0}$. This major contribution leads to many experimental studies aiming at optimizing the shape and dimensions of such "low-profile" VGs (see Betterton et al. 2000; Lin 2002; Angele 2003; Godard and Stanislas 2006; Lögdberg 2006). One of the common results of all these studies is that counter-rotating (CtR) VGs are more efficient than co-rotating VGs. To design some efficient VGs, the relevant parameters appear to be their height $k$, their width $l$, their spanwise spacing $\lambda_{z}$ and their streamwise location from the separation line. Unfortunately, a survey of literature shows a wide range of shapes and values for most of these parameters (see Table 1). The only consensus being about the height of the devices: to limit the induced drag penalty, optimization has been conducted, resulting in a reduction of VGs' height from $k \approx \delta_{0}$ to only a fraction of it (namely $k \leq 0.6 \delta_{0}$ ).

In all those studies, VGs are designed and spaced to promote large energy/circulation CtR vortices with common inflow (see Fig. 1a) downstream of the devices (see the extensive study by Lögdberg 2006). Such vortices push high momentum fluid toward the wall adding the required amount of momentum in the lower energetic regions while there is only a smaller momentum transport from low momentum regions to higher momentum regions.

\subsection{Lift-up and streaky structures}

In laminar shear flows, perturbations consisting in lowenergy counter-rotating streamwise vortices can experience large energy growth through the lift-up effect (see e.g. Moffatt 1967; Landahl 1980). The mechanism is the following: high momentum fluid is pushed toward the wall while low momentum fluid is transported in the outer region of the flow (see Fig. 1b) resulting in streamwiseelongated spanwise modulations of the velocity field called "streaks". The energy growth of these streaks is transient and is strongly related to the non-normal nature of the linearized Navier-Stokes operator (Trefethen et al. 1993). If the shape and the wavelength of the disturbing vortices are optimized, the energy amplification of the resulting optimal streaks can be of order $\mathcal{O}\left(R e^{2}\right)$ (see e.g. Butler and Farrell 1992; Schmid and Henningson 2001). In recent

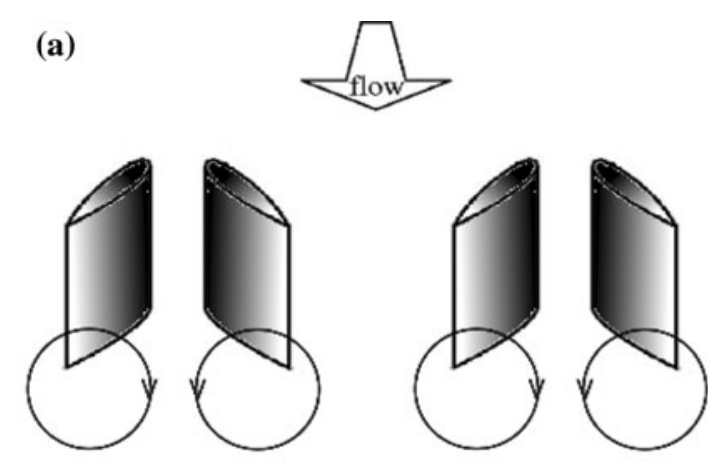

(b)

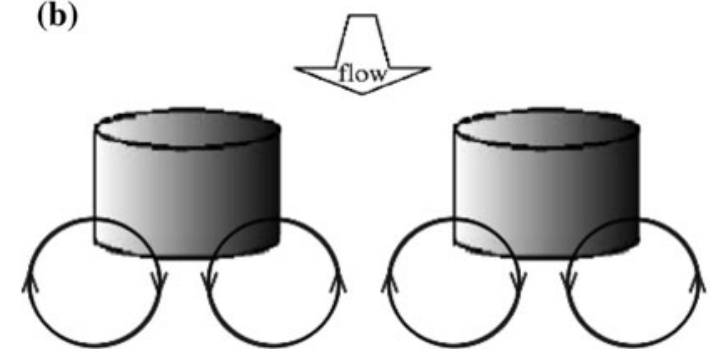

Fig. 1 Sketch of CtR vortices a with common inflow generated by VGs. b interacting with their two neighbors resulting in common inflow and common outflow motions (lift-up effect)

experiments, steady and stable streaks of moderate amplitude have been forced by nearly optimal streamwise vortices generated with cylindrical roughness elements. The resulting streaky flow can be used to manipulate laminar shear flows at leading order and applied to stabilize Tollmien-Schlichting waves (Cossu and Brandt 2002; Fransson et al. 2004) and to delay transition to turbulence (Fransson et al. 2006). Following a similar guideline, Duriez (2009) showed that laminar streaks could also be used to delay separation on a 2D backward-facing ramp in the laminar régime.

Since in many industrial applications, flows are turbulent, an extension of such studies would deal with turbulent shear flows. Optimal perturbations and energy growth sustained by turbulent shear flows have been computed by del Álamo and Jiménez (2006) and Pujals et al. (2009b) for turbulent Poiseuille flows, by Hwang and Cossu (2010) for the turbulent Couette flow and by Cossu et al. (2009) 
for turbulent boundary layers (TBL). Those studies lead to the conclusion that the most amplified perturbations are large-scale streamwise uniform vortices evolving into large-scale coherent streamwise streaks. These structures scale in outer units (i.e. the half-channel width $h$ in the Poiseuille or Couette flow case and the boundary layer thickness $\delta$ in the boundary layer case). In the turbulent boundary layer case, a wide range of spanwise wavelengths ranging from 1 to $30 \delta$ can be largely amplified with an optimal wavelength near $7.6 \delta$.

A recent experimental study aiming at forcing such very large-scale streaks has been conducted by Pujals et al. (2009a, 2010) on a low Reynolds number flat plate TBL $\left(\operatorname{Re}_{\delta^{*}}=U_{e} \delta^{*} / v=1,000\right.$ where $\delta^{*}$ is the displacement thickness). Following the approach of White (2002) and Fransson et al. (2005), arrays of cylindrical roughness elements are used to generate nearly optimal vortices with wavelength ranging from 3 to $12 \delta_{0}$. Similarly to the laminar flow case, the resulting mean flow is spanwise modulated at leading order since finite amplitude very largescale coherent high speed and low speed streaks are observed. The maximum amplitude obtained is of about $13 \%$ of the free-stream velocity $U_{e}$ and is reached around $4-5 \lambda_{z}$ downstream of the array. When compared to the theoretical analysis, the most amplified spanwise spacings in the experiment are in good agreement with predictions of linear stability analysis.

\subsection{Motivations of the present study}

In the present study, large-scale coherent vortices are forced in presence of an adverse pressure gradient due to geometrical constraints. To do so, a generic car model called Ahmed body (Ahmed et al. 1984; Lienhart et al. 2003) is used. The flow around its rear-end presents two salient features: a re-circulation bubble and two vortices originating from the side edges. Since most of the drag force applied to this body is due to pressure drag at the rear-end, a significant drag reduction can be achieved by modifying the flow topology. Previous studies involving active control devices such as blowing or suction slots are reported to be efficient (see Brunn and Nitsche 2006; Roumeas et al. 2009; Leclerc 2008) while there are, to the authors' knowledge, very few studies dealing with "VGslike" passive devices: Beaudoin and Aider (2008) achieved a $25 \%$ drag reduction modifying the streamwise vortices with moving flaps located on the edges of the model; using motorized VGs Aider et al. (2009) obtained a 14\% drag reduction on a modified Ahmed body with a curved rearend. The scope of this study is twofold: (1) to investigate the ability of large-scale coherent streaks to reduce a separation bubble and/or the trailing edge vortices and then to evaluate the amount of drag reduction; (2) to examine the development of the streaks under the influence of an adverse pressure gradient.

In the first part, we describe the facility, the experimental setup and the model used for this study. In Sect. 3, the results are analyzed: we present the macroscopic effect of the controlled flow on time-averaged drag coefficient and relate it to the modification of the flow on the slanted rear-end, then the streaky base flow obtained downstream of the roughness elements is described. Eventually, all those results are discussed and related to previous studies.

\section{Experimental setup}

\subsection{Description of the facility}

All the experiments are carried in PSA in-house facility. It is an Eiffel-type wind tunnel with a rectangular crosssection of $2.1 \mathrm{~m}$ high, $5.2 \mathrm{~m}$ wide and $6 \mathrm{~m}$ long. Velocity can be controlled from 5 up to $55 \mathrm{~m} \mathrm{~s}^{-1}$. Models are fixed on a flat plate placed $500 \mathrm{~mm}$ above the floor. The purpose of this configuration is to control the boundary layer development without any suction device. The flat plate is $3 \mathrm{~m}$ wide and $52 \mathrm{~mm}$ thick. The leading edge is covered with sand-paper to avoid separation in low flow-speed conditions and to promote transition to a turbulent boundary layer. In the following $x, y$ and $z$ are, respectively, the streamwise, normal and spanwise directions. The axis system origin is taken at mid-width on the separation line between the roof and the slanted surface of the model (see Fig. 2).

\subsection{Generic car model and actuators}

The generic car model used here is the one originally described in Ahmed et al. (1984) or Lienhart et al. (2003). The rear part of this model consists in a slanted surface which slant-angle can be modified resulting in very different flow topologies. In the present study, we focus on the $25^{\circ}$ slanted rear-end, the free-stream velocity being set to $U_{e}=20 \mathrm{~m} \mathrm{~s}^{-1}$. This choice of geometry and free-stream velocity leads to a unsteady and three-dimensional flow exhibiting a large separation bubble over the slanted surface along with highly energetic streamwise vortices issuing from the slant side edges. The dimensions and the overall shape of the model are given in Fig. 2. The Reynolds number based on the free-stream velocity $U_{e}$ and the model length $L$ is $R e_{L}=1.35 \times 10^{6}$.

Like in previous studies, cylindrical roughness elements are used to force the large-scale streaks. The dimensions of these elements are of primary importance if we want to generate stable streaks. The relevant parameters are: the height of the cylinders $k$, their diameter $d$ and the spanwise 
Fig. 2 Shape and dimensions of the generic car model used in the present study. From Ahmed et al. (1984). The dimensions are given in millimeters
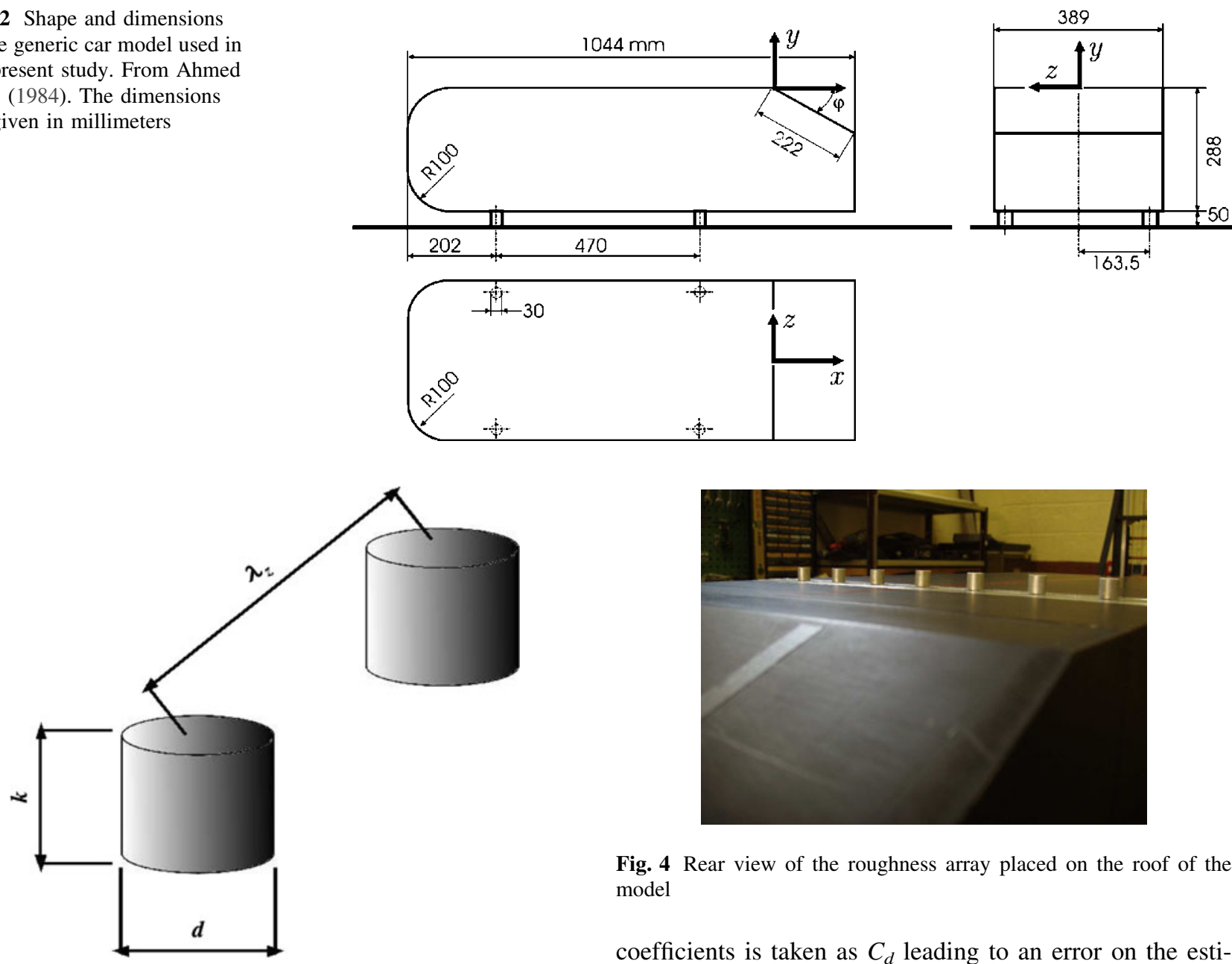

Fig. 3 Shape of the roughness elements and relevant parameters

spacing $\lambda_{z}$. These parameters are illustrated in Fig. 3 while a picture of these roughness elements placed on the roof of the model is displayed in Fig. 4.

\subsection{Experimental measurements}

To evaluate the efficiency of each configuration on the drag force, a six components aerodynamic balance is used. All results hence be expressed as the time-averaged drag coefficient $C_{d}$ :

$C_{d}=\frac{F_{d}}{\frac{1}{2} \rho S U_{e}^{2}}$

where $F_{d}$ is the drag force (i.e. force in the streamwise direction $x), U_{e}$ is the free-stream velocity, and $S$ is the projected surface of the model on the cross-flow plane $(z, y)$. Each measurement consists in five samples of $60 \mathrm{~s}$ at the frequency of $50 \mathrm{~Hz}$. The time-averaged drag coefficient of each sample is computed, and the mean of these five

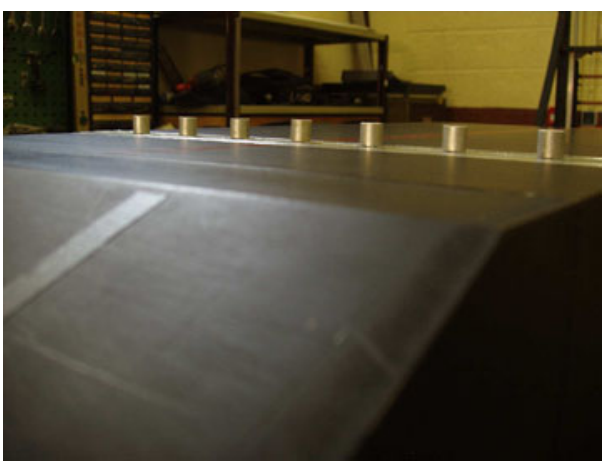

Fig. 4 Rear view of the roughness array placed on the roof of the model

coefficients is taken as $C_{d}$ leading to an error on the estimation lower than $1 \%$.

The influence of large-scale velocity streaks on the static wall pressure distribution and on the rear-end separation is explored using static wall pressure measurements. Pressure sensors are placed all along the symmetry line $z=0$ of the model and on the left half-part of the slanted surface. The wall pressure coefficient is defined as:

$C_{p}=\frac{P-P_{\infty}}{\frac{1}{2} \rho U_{e}^{2}}$

where $P$ is the pressure measured on the model, and $\frac{1}{2} \rho U_{e}^{2}$ and $P_{\infty}$ are, respectively, the dynamic pressure and the static pressure away from the model. The $C_{p}$ estimation is based on the time-averaged value obtained with one sample of $60 \mathrm{~s}$.

The mean velocity is measured using Dantec's flow manager PIV system associated with a $120 \mathrm{~mJ}$ Nd:Yag double cavity laser and a $1,024 \times 1,280$ Hisense 8 bit digital camera. Mean velocity profiles on the roof of the model are measured with a $105 \mathrm{~mm}$ optical lens resulting in $220 \mathrm{~mm} \times 200 \mathrm{~mm}$ planes. A 1,500 pairs of images are acquired to ensure convergence of the mean values and we use $16 \times 8$ pixels interrogation windows with 50 and $75 \%$ 
overlap in respectively the streamwise and normal directions leading to $1.43 \mathrm{~mm}$ and $0.3 \mathrm{~mm}$ spatial resolution. The $(x, y)$ planes around the rear-end are acquired using a $50 \mathrm{~mm}$ lens leading to $400 \mathrm{~mm} \times 400 \mathrm{~mm}$ planes. A 600 pairs of images are used and we perform spatial correlations on $16 \times 16$ interrogation windows with $50 \%$ overlap in each directions resulting in $2.88 \mathrm{~mm} \times 2.88 \mathrm{~mm}$ spatial resolution. The $(x, z)$ planes over the roof and the slanted surface are acquired using the same setup resulting in respectively $315 \mathrm{~mm} \times 253 \mathrm{~mm}$ and $253 \mathrm{~mm} \times 202 \mathrm{~mm}$ planes. Spatial correlations on $16 \times 16$ (resp. $16 \times 8$ ) interrogation windows with $50 \%$ overlap are performed on the 600 pairs of images resulting in $1.6 \mathrm{~mm} \times 1.6 \mathrm{~mm}$ (resp. $1.9 \mathrm{~mm} \times 1.0 \mathrm{~mm}$ ) resolution. The flow is seeded with olive oil droplets of about $300 \mu \mathrm{m}$ diameter.

\section{Baseline configuration and choice of the optimal configuration}

The first part of the present study focuses on the comparison of the effect of various perturbations. On a car model like Ahmed body, since most of the drag is due to pressure drag (see Ahmed et al. 1984), a change in the flow topology (side edge vortices or separation bubble) would have a major impact on the drag coefficient. One way to investigate the effect of a perturbation on the mean flow around the rear-end would be to estimate the resulting drag coefficient.

\subsection{Base configuration without streaks}

For a Reynolds number of $R e_{L}=1.35 \times 10^{6}$, the boundary layer on the roof of the model is fully turbulent. The TBL thickness developing on the roof of the model upstream of the separation line is $\approx 20 \mathrm{~mm}$ and compared to the most widely studied case $R e_{L}=2.7 \times 10^{6}$, the separation over the slanted surface is larger. As the aim of this study is to investigate the effect on separation, the Reynolds number is set to $R e_{L}=1.35 \times 10^{6}$.

The study by Cossu et al. (2009) shows that in the zero pressure gradient turbulent boundary layer case, the most amplified spanwise wavelength is $\lambda_{z} \approx 8 \delta$ and a wide range of wavelengths $\lambda_{z} \in[1 \delta ; 30 \delta]$ can experience large energy growth. According to the first experimental investigation by Pujals et al. (2009a, 2010), very large-scale turbulent streaks can be forced using cylindrical VGs. In this study, the ratio $\lambda_{z} / d$ was constant and set equal to 4 like in previous studies by White (2002) or Fransson et al. (2005). Based on these observations, the constant ratio $\lambda_{z} / d=4$ is also applied in the present study. The height of the cylinders is fixed to $k=12 \mathrm{~mm} \approx 0.6 \delta_{0}$. Even though this value is close to the value used in Fransson et al.
$(2005,2006)$ to delay transition in a laminar boundary layer, the reasons for it are different: in the laminar case, this value was chosen to avoid shedding instabilities that would appear for larger values of $k$ (see section III.C of Fransson et al. 2005) while in the present turbulent case larger values of $k$ would increase the drag penalty induced by the roughness elements themselves.

The selected spanwise spacings are $\lambda_{z}=24,32$ and $48 \mathrm{~mm}$. Scaled with the boundary layer thickness, this results in $\lambda_{z}=1.2,1.6$ and $2.4 \delta_{0}$. Due to the width of the model only "small" spacings are studied; wavelengths comparable to those predicted by Cossu et al. (2009) would result in a very limited number of roughness elements. Table 2 summarizes those configurations. Given these parameters, various positions all along the roof of the model are studied with configurations $\mathrm{A}$ to $\mathrm{C}$, the goal of this first step being to determine for each roughness array the optimal streamwise position and then to highlight the global optimal configuration.

\subsection{Effect on drag force}

The efficiency of each roughness array is quantified regarding the time-averaged drag coefficient $C_{d}$ reduction defined as $\Delta C_{d}=2 \cdot \frac{C_{d}-C_{d}^{0}}{C_{d}+C_{d}^{0}}$, where $C_{d}^{0}$ denotes the timeaveraged drag coefficient in the undisturbed case. In Fig. 5, the drag reduction obtained with each configuration is plotted against the distance from the separation line $x_{0}$ expressed in meters (Fig. 5a) and scaled on the wavelength of the perturbation $\lambda_{z}$ (Fig. 5b). As shown in Fig. 5a, the drag decreases as the roughness array is moved toward the separation line until it reaches a minimum value for an optimal streamwise position denoted $x_{0}^{\text {opt }}$ (note that the value $x_{0}^{\text {opt }}$ depends on $\lambda_{z}$ ). Then, when the array is placed downstream of $x_{0}^{\mathrm{opt}}$, the drag starts to increase. However, when scaled on the spanwise spacing $\lambda_{z}$, the optimal streamwise position is always in the range $4-5 \lambda_{z}$ upstream of the separation line. The maximum drag reduction achieved here is of about $10 \%$.

\section{Analysis of the optimal drag-reducing configuration}

Substantial drag reduction is obtained using every optimal perturbations tested here. Nevertheless, the reason for it is

Table 2 Description of the configurations analyzed in this study

\begin{tabular}{llclll}
\hline Configuration & $\lambda_{z}(\mathrm{~mm})$ & $d(\mathrm{~mm})$ & $\lambda_{z} / \delta_{0}$ & $\lambda_{z} / d$ & $k / \delta_{0}$ \\
\hline A & 24 & 6 & 1.2 & 4 & 0.6 \\
B & 32 & 8 & 1.6 & 4 & 0.6 \\
C & 48 & 12 & 2.4 & 4 & 0.6 \\
\hline
\end{tabular}



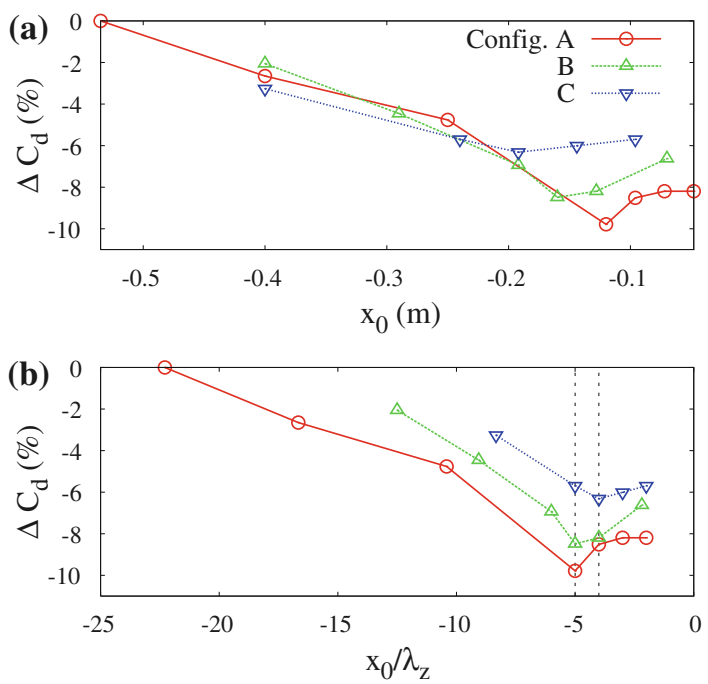

Fig. 5 Drag reduction for each tested wavelength as a function of the distance from the separation line a expressed in meters, $\mathbf{b}$ scaled on the spanwise spacing $\lambda_{z}$. The two vertical dashed lines on $\mathbf{b}$ are $x_{0}=-5 \lambda_{z}$ and $x_{0}=-4 \lambda_{z}$

still a not addressed question. Here, we focus on the optimal drag-reducing configuration, which is configuration $\mathrm{A}$ with $x_{0}=-5 \lambda_{z}$. In order to find out how the topology of the flow around the rear-end is modified (are the re-circulation bubble and/or streamwise vortices modified?), PIV planes both on the symmetry plane $z=0$ and parallel to the slanted surface are acquired, and static wall pressure is measured on the slanted surface.

\subsection{Mean flow velocity field and topology around} the rear-end

In Fig. 6, the streamwise velocity $U / U_{e}$ measured in the symmetry plane around the rear-end is plotted for the uncontrolled case (Fig. 6a) and for the controlled case with configuration A at $x_{0}=x_{0}^{\text {opt }}$ (Fig. 6b). The flow is from left to right. The uncontrolled flow presents a thick shear layer originating from the upper side of the slanted surface. The curvature of the whole shear layer and the dark blue spot between the surface and the layer indicates a re-circulation bubble. When control is applied, the shear layer becomes thinner and its curvature is highly reduced. This behavior suggests that the re-circulation bubble has been suppressed or at least highly reduced.

To confirm this result, the near-wall PIV measures on $(x, z)$ planes are carried out at $Y / k=0.08$ (corresponding to $Y=1 \mathrm{~mm}$ from the slanted surface). The resulting streamwise velocity fields (colored contours) as well as the time-averaged streamlines (vectors field) are reported in Fig. 7 for the two cases discussed previously. The flow is from top to bottom. The uncontrolled flow (Fig. 7a) presents the common features discussed in 1.3. The re-circulation bubble is clearly apparent since a backward flow region is observed for roughly $0.01 \mathrm{~m} \leq x \leq 0.15 \mathrm{~m}$ along the symmetry line $z=0 \mathrm{~m}$. The footprint of one streamwise conical vortex is also observed on the right part of the figure. The controlled flow displayed on Fig. 7b confirms the previous suggestion: the re-circulation bubble is suppressed since the streamlines present no evidence of backward flow. Nevertheless, no noticeable influence on the streamwise vortices can be observed at this distance from the wall.

Both the re-circulation bubble and side edge vortices have a strong influence on the pressure distribution on the slanted surface. Any modification on the topology of the flow would implies a change in this distribution. In order to evaluate it, static wall pressure measurements have been carried out.

\subsection{Wall pressure distribution on the slanted surface}

Figure 8 represents the wall pressure coefficient $C_{p}$ for the uncontrolled reference case (Fig. 8a) and for the controlled studied in the previous section (Fig. 8b). On both figures, the flow is from top to bottom. The reference case exhibits two regions where the pressure coefficient is locally lower. The first one can be seen on the left part of the figure. It corresponds to the footprints of the side edge vortex originating from the trailing edge of the model described by Lienhart et al. (2003). The second region spans almost the whole model's width and extends from the separation line
Fig. 6 Time-averaged streamwise velocity $U / U_{e}$ around the rear-end in the symmetry plane using PIV. The flow is from left to right. a Uncontrolled case. b Optimally controlled case with configuration $\mathrm{A}$ at $x_{0}=x_{0}^{\text {opt }}$
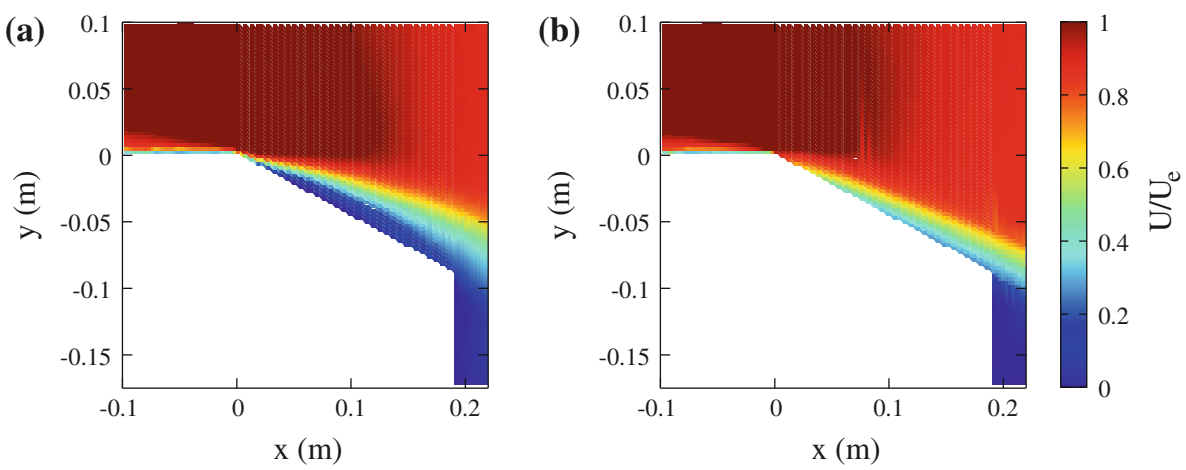
Fig. 7 Time-averaged

streamwise velocity $Y / k=0.08$ above the slanted surface using near-wall PIV. The line $x=0$ is the beginning of the slanted surface. The flow is from top to bottom. a Uncontrolled case. b Optimally controlled case with the same configuration as in Fig. $6 b$

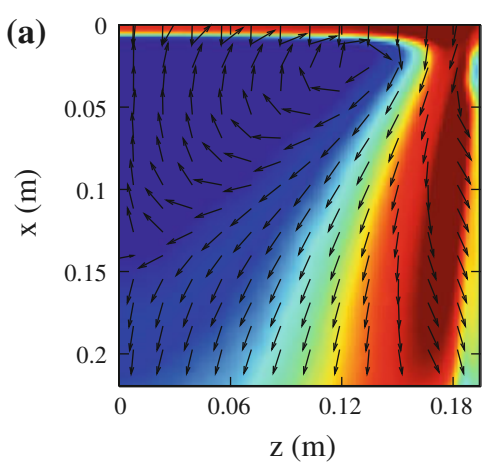

$\mathrm{z}(\mathrm{m})$

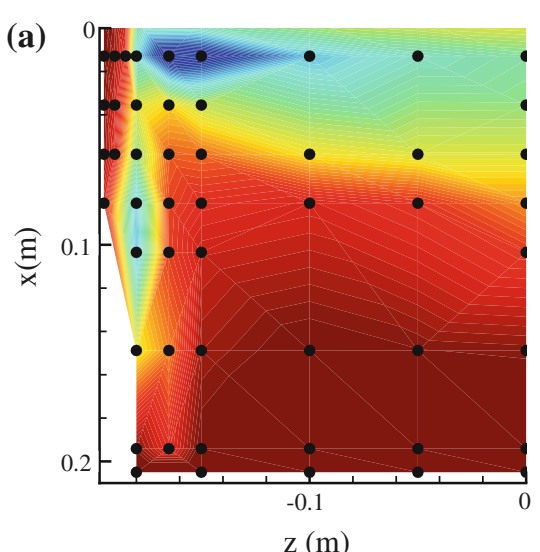

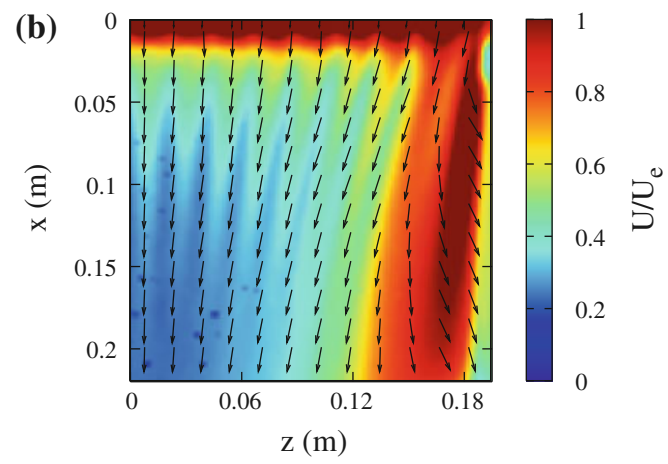

(b)

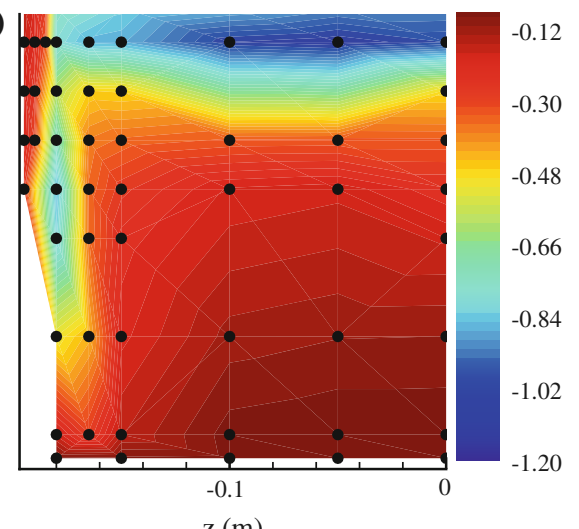

$\mathrm{z}(\mathrm{m})$
Fig. 8 Wall pressure coefficient $C_{p}$ on the left halfpart of the slanted surface for $R e_{L}=1.35 \times 10^{6}$. The line $x=0$ is the beginning of the slanted surface. The flow is from top to bottom. a Reference flow without control. b Flow controlled with same configuration as in Fig. 6. The symmetry plane is on $z=0$, the upper side edge is on $x=0$. The black circles on each plot show the position of the pressure sensors $x=0 \mathrm{~m}$ to $x \approx 0.15 \mathrm{~m}$. As we move along the streamwise direction, the pressure coefficient slowly recovers. This second area corresponds to the separation bubble. It can be seen from the $C_{p}$ distribution associated with the controlled flow that the pressure is even lower in the region just downstream of the separation line. This behavior is due to the acceleration of the attached flow around the sharp upper edge of the slanted surface. Nevertheless, the reduced/ vanished re-circulation bubble induces a pressure recovery far upstream than in the uncontrolled case. The effect of the roughness array on the streamwise vortices seems negligible since the footprints observed on (a) and (b) are extremely similar.

The $C_{p}$ obtained with configurations $\mathrm{A}, \mathrm{B}$ and $\mathrm{C}$ along the symmetry line $z=0$ is displayed in Fig. 9 along with the uncontrolled reference. When control is applied, the $C_{p}$ distributions are very similar and all the curves collapse on a single one. Compared to the uncontrolled case, the pressure recovery is quicker. This suggests that the effect on the separation bubble is also observed for the other configurations (PIV measurements not displayed here confirm this result). The discrepancy on drag reduction efficiency presented in Sect. 3.2 would then be due to the drag penalty induced by the larger cylinders used in those cases. The only slight difference between the configurations $\mathrm{A}, \mathrm{B}$ and $\mathrm{C}$ comes from the low-pressure region just downstream of the upper edge.

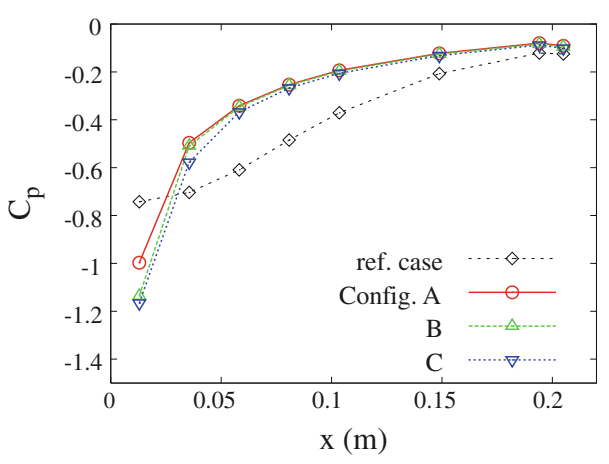

Fig. 9 Wall pressure coefficient $C_{p}$ measured for the uncontrolled reference case and the configurations $A, B$ and $C$ with roughness arrays placed at $x=x_{0}^{\mathrm{opt}}$ along the symmetry line $z=0$

In recent experiments, substantial drag reduction had been achieved by prolongating the separation with flaps (Beaudoin and Aider 2008) or VGs (Aider et al. 2009). As explained in those references, prolongating the separation permits to highly reduce and even suppress the side edges streamwise vortices. Hence, even though the separation is promoted, the pressure loss due to those streamwise vortices is reduced in such a way that the sum of both contributions (vortices and separation) is decreased. On the contrary, in the present experiments, the streaky incoming flow remains attached over the slanted surface (hence increasing the pressure distribution over this surface) while 
the edge vortices do not experience major modifications. The balance between pressures felt by the front-end and the rear-end is modified, and the total drag of the model is reduced.

\subsection{Topology of the flow on the roof}

The scope of the next section is to focus on the structures generated by the roughness elements array in order to provide an explanation for this separation delay.

Figure 10 shows a visualization of the mean flow over the roof measured downstream of the $d=6 \mathrm{~mm}$ (Fig. 10a) cylinders array. The flow is from left to right, and the cylinders (represented by the white circles on the left part of the figure) are located on the left part of the figures. The velocity is scaled on the free-stream velocity $U_{e}$ and plotted versus the spanwise and streamwise directions both scaled on the spacing $\lambda_{z}$. The mean flow is clearly spanwise modulated and alternating patterns of high speed (hot contours) and low speed (cold contour) streaks are observed. These velocity streaks are observable about $x=1 \lambda_{z}$ downstream of the cylinders array up to the separation line at $x=0 \lambda_{z}$.

Like in previous studies involving laminar flows (Fransson et al. 2005) or the flat plate TBL (Pujals et al. 2009a, 2010), the mechanism of streaks generation is a three-stage process: first, streamwise vortices are generated by the roughness elements; second, those vortices induce streaks by lift-up effect (the amplitude of the streaks grows while the vortices decay) then the streaks decay. The very first stage of this process can be observed in Fig. 10 from $x=-5 \lambda_{z}$ to roughly $x=-4 \lambda_{z}$. However, a measure of the amplitude of the streaks has to be defined in order to study the two other stages. Various measures of the streaks amplitude can be found in literature: the kinetic energy

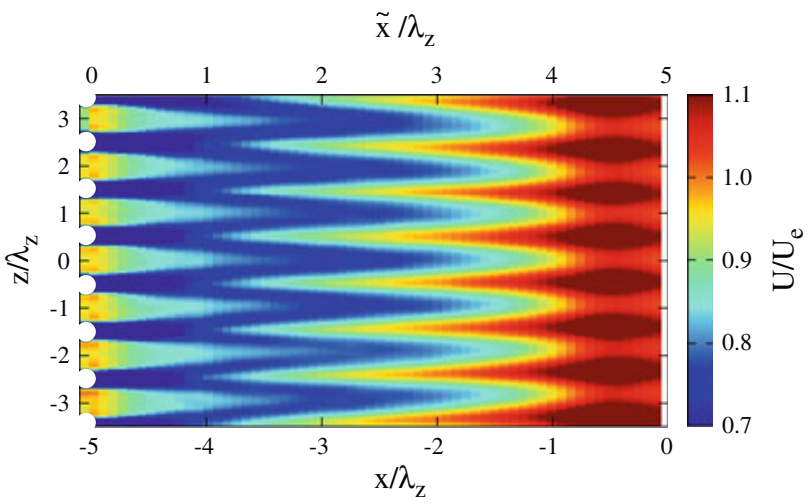

Fig. 10 Large coherent velocity streaks forced on the roof of the model at $Y / k=0.5(Y=6 \mathrm{~mm})$ from the wall. The flow is from left to right. Same configuration as in Fig. 6. Time- averaged streamwise velocity $U / U_{e}$ as a function of the streamwise and spanwise directions $x / \lambda_{z}$ and $z / \lambda_{z}$ criterion or the min-max definition used by Andersson et al. (2001) for instance. In the present case, the experimental apparatus does not permit to quantify the kinetic energy contained in the vortices induced by the roughness elements. Consequently, the amplitude is estimated using a local min-max criterion:

$\widehat{A_{\mathrm{st}}}(\tilde{x}, Y / k=0.5)=\frac{U_{\mathrm{hss}}(\tilde{x}, Y / k=0.5)-U_{\mathrm{lss}}(\tilde{x}, Y / k=0.5)}{2 U_{e}}$

where $U_{\mathrm{hss}}$ and $U_{\mathrm{lss}}$ are respectively the streamwise velocity in a high speed and low speed streak. A more accurate value is then obtained performing a sliding averaging over the width of the window.

The amplitude $\widehat{A_{\mathrm{st}}}(\tilde{x}, Y / k=0.5)$ of the streaks arising with configuration $\mathrm{A}$ is plotted against the normalized distance from the cylinder array $\tilde{x} / \lambda_{z}$ in Fig. 11 . The streaks experience an algebraic growth period with a maximum observed amplitude close around $8 \%$ of the freestream velocity $U_{e}$. The maximum amplitude is reached between 2.5 and 3.5 wavelengths downstream of the array. This behavior is slightly different from the zero pressure gradient case (see Pujals et al. 2010). This is probably due to the adverse pressure gradient and the vicinity of the separation line.

\section{Summary and discussion}

The potential of a separation control strategy based on large-scale coherent streaks is experimentally investigated. Such streaks are generated by means of an array of cylindrical roughness elements on a highly three-dimensional flow subject to an adverse pressure gradient due to geometrical constraints. The geometry chosen here is the $25^{\circ}$ slanted rear-end of the Ahmed body at Reynolds number $R e_{L}=1.35 \times 10^{6}$.

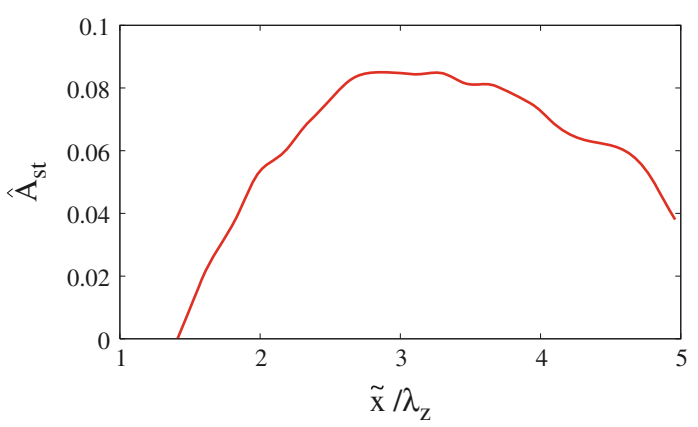

Fig. 11 Estimated streaks' finite amplitude $\widehat{A_{\text {st }}}(x, Y / k=0.5)$ for configuration $\mathrm{A}$ as a function of the distance from the cylinders array. The distance is scaled with the spanwise spacing $\tilde{x} / \lambda_{z}$ 
Various configurations of spanwise spacings and streamwise locations on the roof are investigated measuring their influence on total drag reduction. The main results found are as follows:

- Substantial drag reduction is obtained for all sets of wavelength and streamwise location.

- For all the configurations tested, the streamwise position leading to the maximum drag reduction is $4-5 \lambda_{z}$ upstream of the separation line. The overall maximum total drag reduction measured here is about $10 \%$.

- PIV as well as static pressure measurements on the slanted surface prove that the drag reduction is due to the suppression to the re-circulation bubble.

The analysis of the controlled flow on the roof confirms that the array of cylinders generates streamwise vortices, which lead to coherent streamwise streaks through the liftup effect. Similarly to previous investigations on flat plate turbulent boundary layers, those streaks are stable and of finite amplitude. The maximum amplitude obtained facing the adverse pressure gradient is near $9 \%$ of the free-stream velocity. The resulting streaky base flow seems responsible for the modification of the flow topology further downstream since traces of the streaky flow are still observable on the velocity fields over the slanted surface.

In the present study, for wind-tunnel availability reasons, we have not attempted to find the value of the minimum streak amplitude necessary to suppress the separation. Probably, the total drag reduction could be improved by finding such a minimum value because lower streaks amplitudes would be obtained by reducing the height $k$ of the roughness elements (see White 2002; Fransson et al. 2004 for the laminar case) and therefore reducing the drag penalty induced by the roughness elements themselves.

The potential of cylindrical VGs to delay the separation of a laminar flow over a smooth 2D ramp has been experimentally demonstrated by Duriez (2009). In this study, the diameter and height of the cylinders are kept constant while their spanwise spacing is set to $\lambda_{z}=2,3$ and $4 d$. The roughness array is located at $x_{V G}=12.5 d$ upstream of the ramp resulting in $x_{V G} \in\left[3 \lambda_{z}, 6 \lambda_{z}\right]$ depending on the wavelength. According to this study, the larger wavelength $\lambda_{z}=3$ and $4 d$ are the most effective in delaying the separation. This delay is due to the averaged influence of the spanwise modulation of the base flow. The approach followed by Duriez (2009) is different from the one proposed in the present paper since it applies to a laminar base flow. Furthermore as here we keep the ratio $\lambda_{z} / d=4$ constant, the streaky base flows are similar like in the study of Fransson et al. (2005), and the mechanism involved in the streaks' growth is then the same regardless of the configuration.
It is, finally, important to emphasize the differences between the proposed technique, based on the nearly optimal forcing of streaks, and related passive techniques in turbulent flows. As an example, we consider the very interesting study of Park et al. (2007) who considered the flow over a backward-facing step and showed that a $51 \%$ reduction of the reattachment length can be obtained using rectangular tabs located at the edge of the step. Those tabs induce two main effects on the mean flow: a spanwise modulation of the streamwise velocity profile and strong streamwise vortices issued from the tabs. Park et al. (2007) ascribe the bubble reduction to the action of the 'flying' streamwise vortices issued from the tabs and that work by pairs to create a vertical mixing on the bubble edge (this behavior is the same when dealing with "regular VGs", see e.g. Godard and Stanislas 2006). On the contrary, in the present study, the separation delay must be attributed only to the streaks (the spanwise modulation of the streamwise velocity) because streamwise vortices have virtually disappeared at the position of maximum streak amplitude, which is a typical feature of the lift-up effect. This, again, underlines the two-stage nature of the technique we suggest. The first stage, localized well upstream of the edge, is the forcing of nearly optimal low-energy streamwise vortices. The second stage, localized downstream, consists in the separation delay induced by the important spanwise modulation of the mean flow associated with large-amplitude coherent streaks. These two stages are coupled by the lift-up effect by which the forced low-energy vortices induce large-amplitude coherent streaks extracting energy from the mean flow itself.

Acknowledgments We acknowledge S. Kerling, D. Lancelle, G. Hulin for their contribution to wind tunnel measurements and A. Garcia, G. Peignée for the conception of the roughness elements.

\section{References}

Ahmed SR, Ramm R, Faltin G (1984) Some salient features of the time-averaged ground vehicle wake. SAE 840300

Aider JL, Beaudoin JF, Wesfreid JE (2009) Drag and lift reduction of a $3 \mathrm{~d}$ bluff body using active vortex generators. Experiments in Fluids pp1-19

Andersson P, Brandt L, Bottaro A, Henningson D (2001) On the breakdown of boundary layers streaks. J Fluid Mech 428:29-60

Angele K (2003) Experimental studies of turbulent boundary layer separation and control. $\mathrm{PhD}$ thesis, KTH, Mechanics

Angele K, Grewe F (2007) Instantaneous behavior of streamwise vortices for turbulent boundary layer separation control. J Fluids Eng 129:226-235

Beaudoin JF, Aider JL (2008) Drag and lift reduction of 3d bluff body using flaps. Exp Fluids 44:491-501

Betterton J, Hackett K, Ashill P, Wilson M, Woodcock I, Tilman C, Langan K (2000) Laser doppler anemometry investigation on sub boundary layer vortex generators for flow control. in: 10th symposium on application of laser techniques to fluid mechanics, Lisbon, pp 10-12 
Brunn A, Nitsche W (2006) Active control of turbulent separated flow over slanted surfaces. Int J Heat Fluid Flow 27:748-755

Butler KM, Farrell BF (1992) Three-dimensional optimal perturbations in viscous shear flow. Phys Fluids A 4:1637-1650

Cossu C, Brandt L (2002) Stabilization of Tollmien-Schlichting waves by finite amplitude optimal streaks in the Blasius boundary layer. Phys Fluids 14:L57-L60

Cossu C, Pujals G, Depardon S (2009) Optimal transient growth and very large scale structures in turbulent boundary layers. J Fluid Mech 619:79-94

del Álamo JC, Jiménez J (2006) Linear energy amplification in turbulent channels. J Fluid Mech 559:205-213

Duriez T (2009) Application des générateurs de vortex au contrôle d'écoulements décollés. PhD thesis, Université Paris Diderot

Duriez T, Aider JL, Wesfreid JE (2006) Base flow modification by streamwise vortices. application to the control of separated flows. ASME Technical Paper FEDSM2006-98541, Control of separated flows, Miami, USA

Fransson J, Brandt L, Talamelli A, Cossu C (2004) Experimental and theoretical investigation of the non-modal growth of steady streaks in a flat plate boundary layer. Phys Fluids 16:3627-3638

Fransson J, Brandt L, Talamelli A, Cossu C (2005) Experimental study of the stabilisation of Tollmien-Schlichting waves by finite amplitude streaks. Phys Fluids 17:54-110

Fransson J, Talamelli A, Brandt L, Cossu C (2006) Delaying transition to turbulence by a passive mechanism. Phys Rev Lett 96:064,501

Godard G, Stanislas M (2006) Control of a decelerating boundary layer. part 1: optimization of passive vortex generators. Aero Sci Tech 10:181-191

Hwang Y, Cossu C (2010) Amplification of coherent streaks in the turbulent Couette flow: an input-output analysis at low Reynolds number. J Fluid Mech 643:333-348

Landahl MT (1980) A note on an algebraic instability of inviscid parallel shear flows. J Fluid Mech 98:243

Leclerc C (2008) Réduction de la traînée d'un véhicule automobile simplifié à l'aide du contrôle actif par jet synthétique. $\mathrm{PhD}$ thesis, Institut National Polytechnique de Toulouse (INP), Toulouse

Lienhart H, Stoots C, Becker S (2003) Flow and turbulence structure in the wake of a simplified car model. SAE Trans 112(6):785796

Lin JC (1999) Control of turbulent boundary layer separation using micro-vortex generators. AIAA paper 99-3404
Lin JC (2002) Review of research on low-profile vortex generators to control boundary layer separation. Porg Aerosp Sci 38(45):389-420

Lögdberg O (2006) Vortex generators and turbulent boundary layer separation control. Master's thesis, Royal Insitute of Technology (KTH), Stockholm

Moffatt HK (1967) The interaction of turbulence with strong wind shear. In: Yaglom A, Tatarsky VI (eds) Proceeding URSI-IUGG Coloq. on Atoms. Turbulence and Radio Wave Propag., Nauka, Moscow, pp 139-154

Park H, Jeon W, Choi H, Yoo J (2007) Mixing enhancement behind a backward-facing step using tabs. Phys Fluids 19:103-105

Pastoor M, Henning L, Noack B, King R, Tadmor G (2008) Feedback shear layer control for bluff body drag reduction. J Fluid Mech 608:161-196

Pujals G, Cossu C, Depardon S (2009a) Optimal transient growth and very large-scale structures in zero-pressure gradient turbulent boundary layers. In: sixth international symposium on turbulence and shear flow phenomena, Seoul, Korea, 22-24 June 2009

Pujals G, García-Villalba M, Cossu C, Depardon S (2009b) A note on optimal transient growth in turbulent channel flows. Phys Fluids 21:015,109

Pujals G, Cossu C, Depardon S (2010) Forcing large-scale coherent streaks in a zero pressure gradient turbulent boundary layer. Submitted to Journal of Turbulence

Rao DM, Kariya TT (1988) Boundary-layer submerged vortex generators for separation control - an exploratory study. In: AIAA, ASME, SIAM and APS National Fluid Dynamics Congress, 1 st, Cincinnati, OH; UNITED STATE, Washington, DC, American Institute of Aeronautics and Astronautics, pp 839-846

Roumeas M, Gillieron P, Kourta A (2009) Drag reduction by flow separation control on a car after body. Int J Numer Meth Fluids 60(11):1222-1240

Schmid PJ, Henningson DS (2001) Stability and transition in shear flows. Springer, New York

Taylor H (1947) The elimination of diffuser separation by vortex generators. Report No R-4012-3, United Aircraft Corporation

Trefethen LN, Trefethen AE, Reddy SC, Driscoll TA (1993) A new direction in hydrodynamic stability: beyond eigenvalues. Science 261:578-584

White EB (2002) Transient growth of stationary disturbances in a flat plate boundary layer. Phys Fluids 14:4429-4439 